\title{
Médiévales
}

Langues, Textes, Histoire

76 | printemps 2019

Du nouveau en archives

\section{Agostino PARAVICINI BAGLIANI dir., The Impact of Arabic Sciences in Europe and Asia}

Florence, Sismel/Edizioni del Galluzzo (Micrologus, XXIV), 2016, vi-504 p.

\section{Marc Moyon}

\section{(2) OpenEdition}

1 Journals

\section{Édition électronique}

URL : https://journals.openedition.org/medievales/10156

DOI : 10.4000/medievales.10156

ISSN : $1777-5892$

\section{Éditeur}

Presses universitaires de Vincennes

\section{Édition imprimée}

Date de publication : 5 septembre 2019

Pagination : $177-182$

ISBN : 978-2-37924-028-7

ISSN : 0751-2708

\section{Référence électronique}

Marc Moyon, "Agostino paravicin BaglianI dir., The Impact of Arabic Sciences in Europe and Asia », Médiévales [En ligne], 76 I printemps 2019, mis en ligne le 05 septembre 2019, consulté le 22 avril 2022. URL : http://journals.openedition.org/medievales/10156 ; DOI : https://doi.org/10.4000/ medievales. 10156

Ce document a été généré automatiquement le 22 avril 2022.

Tous droits réservés 


\section{Agostino PARAVICINI BAGLIANI dir., The Impact of Arabic Sciences in Europe and Asia}

Florence, Sismel/Edizioni del Galluzzo (Micrologus, XXIV), 2016, vi-504 p.

Marc Moyon

\section{RÉFÉRENCE}

Agostino PARAVICINI BAGLIANI dir., The Impact of Arabic Sciences in Europe and Asia, Florence, Sismel/Edizioni del Galluzzo (Micrologus, XXIV), 2016, vi-504 p.

1 Le rôle de l'appropriation des sciences arabes dans le développement des sciences en Europe et en Asie a fait débat et fait régulièrement débat, en particulier pour l'Europe, où le parti-pris idéologique n'est malheureusement pas toujours aussi absent qu'il devrait l'être. Il n'est sans doute pas inutile de rappeler ici que l'expression «science arabe » renvoie à la science élaborée dans les pays d'Islam, écrite en arabe tout au long de la période dite classique ( $\mathrm{VIII}^{\mathrm{e}}-\mathrm{XVI}^{\mathrm{e}}$ siècles). Les sciences arabes regroupent donc toute la production des auteurs s'exprimant en langue arabe, d'origines et de confessions diverses.

2 L'historiographie est riche de nombreux articles spécialisés et de plusieurs ouvrages de synthèse qui permettent d'évaluer ou de réévaluer, à la lumière de nouvelles connaissances, l'impact des savoirs ou des pratiques des pays d'Islam pour un champ disciplinaire ou encore la dette d'un auteur envers un ou plusieurs de ses prédécesseurs de langue arabe. Nous pouvons maintenant considérer l'ouvrage sous recension comme une référence pour ce genre d'étude. Il est le résultat éditorial de la conférence internationale The Impact of Arabic Sources on Divination and the Practical Sciences in Europe and Asia qui s'est tenue à Erlangen (Allemagne) en janvier 2014. L'ouvrage est organisé autour de trois parties principales qui, sans justification, peuvent sembler relativement 
arbitraires : elles concernent tour à tour "les sciences pratiques", "la divination, la magie et l'astrologie », et enfin " la réception et l'organisation de la connaissance ». Ces trois parties suivent deux chapitres introductifs et précèdent deux chapitres de conclusion. Enfin, l'ouvrage est complété par deux précieux index : celui des noms et des lieux (p. 483-501) et celui des manuscrits (p. 503-504).

Charles Burnett signe le premier chapitre introductif (p.3-11). Il y livre une étude terminologique du «juger» (iudicare) et du «faire» (facere/operare) dans les textes latins et arabes d'astrologie, discipline apparaissant littéralement comme la «science des jugements " ('ilm al-ahkām en arabe et scientia de iudiciis en latin). À partir de cette étude, C. Burnett établit les différences entre les jugements des astrologues et ceux des étoiles elles-mêmes. Danielle Jacquard rédige le second chapitre de l'introduction en rappelant quelques généralités pour quiconque s'intéresserait aux sources des Latins (p. 13-21). En particulier, si les sciences arabes sont les sources incontestables des sciences latines, leurs rapports de dépendance envers les sciences grecques demandent toujours à être finement analysés. En effet, les Arabes et les Grecs étant considérés, au Moyen Âge, comme des autorités, la part des uns et celle des autres ne sont souvent pas bien évaluées et ce, déjà, par les médiévaux eux-mêmes. Les textes grecs, arabes et gréco-arabes sont traduits, commentés, corrigés ou transformés au gré de leur diffusion. En outre, il ne faut pas non plus oublier, et Danielle Jacquard nous le remémore fort justement, que les influences d'une tradition vers une autre ne passent pas nécessairement par les seules traductions.

4 La première partie, sur les "sciences pratiques", regroupe cinq contributions ayant trait à la médecine, l'alchimie, l'algèbre et la mécanique. D'abord, Joël Chandelier s'intéresse au concept de maladie chronique, par opposition à celui de maladie aiguë, dans sa transmission des Grecs aux Arabes et des Arabes aux Latins (p. 25-42). Il s'agit avant tout de décrire ce que la médecine arabe développée par al-Majūsī ( $\mathrm{x}^{\mathrm{e}}$ siècle) et Ibn Sīnā/Avicenne (m. 1037) a d'original par rapport à la médecine grecque, surtout par rapport à celle des méthodistes. J. Chandelier peut alors mieux estimer ce que les Latins, dans leurs traités et leurs pratiques, doivent à la médecine arabe : «ce n'est pas par l'invention d'un concept nouveau que les Arabes ont durablement marqué la discipline, mais par un subtil réarrangement de la matière ancienne provoquant à terme un véritable changement de perspective - et ce, très certainement, sans même que les auteurs latins ne s'en aperçoivent» (p. 42). Toujours en médecine, Michael Rogers Mc Vaugh s'interroge sur les raisons de la faculté de médecine de Montpellier pour intégrer dans leur programme d'étude, dès les premières années du XIV siècle, le médecin persan Razi/Rhazès comme une autorité, au même titre qu'Ibn Sīnā (p. 43-72). Pour cela, M. R. Mc Vaugh analyse les écrits de trois auteurs du xive siècle qui ont étudié à la faculté de Montpellier et enseigné ou pratiqué la médecine non loin de là : Gérard de Solo (m. ca 1350), Guy de Chauliac (m. 1368) et Jean de Tournemire (m. 1396), dont il édite six folios d'un Regimen sanitatis rédigé en 1373 (p. 67-71). Il montre que Razi est apprécié non seulement pour tous les détails qu'il donne dans la description des traitements envisagés, mais aussi comme modèle pour la pratique médicale. Il apparaît comme une vraie alternative à l'académisme d'Ibn Sīnā. Avec Michela Pereira est discutée une autre science pratique introduite en Europe à partir des traductions arabo-latines des XII et XIII ${ }^{\mathrm{e}}$ siècles : l'alchimie (al-kimyyâ) (p. 73-94). M. Pereira débute son chapitre en détaillant les premières traces de l'alchimie en Europe médiévale, avant de s'intéresser précisément à l'élixir (al-iksir), cette substance capable d'améliorer la vie 
humaine et de réduire la souffrance de l'humanité (p. 80), et à son alchimie (différente de la tradition alchimique de l'or de transmutation). L'argumentation de M. Pereira s'appuie sur les travaux d'Arnaud de Villeneuve, de Ramon Lull et de John Dastin, tous du XIV ${ }^{e}$ siècle, qui, après Roger Bacon, considèrent explicitement l'élixir comme produit de guérison du corps humain. Le chapitre suivant est consacré à l'algèbre (al-jabr) qui, dans la Risāla fi aqsām al-'ulūm al-'aqliyya [Épître sur les parties des sciences intellectuelles] d'Ibn Sīnā, devient un art faisant partie de la science des nombres ('ilm al-'adad). Ahmed Djebbar revient sur le développement de la discipline à partir du Mukhtașar fi hisāb al-jabr wa-l- muqābala [Abrégé du calcul par la restauration et la comparaison] d'al-Khwārizmī (m. 850) aussi bien en Orient qu'en Occident musulmans (p. 95-119). Cet état des lieux de la production algébrique à l'intérieur des frontières des pays d'Islam lui permet de mieux appréhender la circulation des textes arabes et leur impact sur la production médiévale latine ${ }^{1}$ et hébraïque. A. Djebbar ne limite pas son étude au seul Moyen Âge central, mais poursuit vers les traditions vernaculaires du Moyen Âge tardif avec le cas des traditions italienne, française et allemande. Enfin, Mohammed Abattouy rédige le dernier chapitre consacré aux sciences pratiques avec son étude détaillée du corpus des textes rédigés par al-Isfizāaī ( $\mathrm{XI}^{\mathrm{e}}$-XII ${ }^{\mathrm{e}}$ siècles) sur la mécanique (p. 121-169). Trop longtemps ignoré, ce corpus représente une importante source pour la mécanique arabe, aussi bien dans ses dimensions théoriques que pratiques. En particulier, pour M. Abattouy, l'étude approfondie de ce corpus met en évidence une transformation de la mécanique arabe, au sein de laquelle une science des poids ('ilm al-athqāl en arabe et scientia de ponderibus en latin) émerge comme une branche indépendante et séparée de la science des procédés ingénieux ('ilm al-hiyal/ scientia de ingeniis ou ingeniorum scientia).

5 La deuxième partie de cet ouvrage est centrée sur la divination, la magie et l'astrologie, avec six chapitres. David Juste ouvre cette partie avec un chapitre dans lequel il souhaite mesurer l'impact de la transmission des sources astrologiques arabes en quantifiant leur poids dans le corpus manuscrit aujourd'hui disponible dans les bibliothèques (p. 173-194). Le texte de D. Juste est un modèle méthodologique pour l'étude quantitative d'un corpus ${ }^{2}$. Il a listé les cinquante textes astrologiques les plus populaires disponibles en latin et datés d'avant 1500 . Il en établit une classification selon l'origine (arabe, grecque, hébreu, latin), l'auteur, la date de production et le sujet. La tâche est fastidieuse, mais les résultats sont sans appel: entre autres, vingt-huit textes sont d'origine arabe et représentent plus de la moitié de l'ensemble des copies disponibles (p. 179). Le chapitre suivant, signé par Jean-Patrice Boudet, propose une réflexion sur la manière de considérer les comètes au Moyen Âge (p. 195-226). Pour cela, J.-P. Boudet étudie le Centiloquium et le De cometis, deux textes faussement attribués à Ptolémée et traduits à partir de l'arabe au XII siècle. La contribution est très érudite et offre aux lecteurs de nombreux extraits sur les comètes (soit à partir d'éditions existantes, soit à partir des manuscrits lorsqu'aucune édition n'existe). Centrale est ici la question des sources. En effet, sont-elles uniquement arabes, grecques et arabes ou encore arabes et latines? Difficile de répondre à cette question dans ce cas précis où aucun manuscrit arabe du De cometis n'a encore été découvert. J.P. Boudet termine enfin son étude sur l'influence, jusqu'à la Renaissance, de ce court traité à propos des comètes. C'est la magie, spécialité de Nicolas Weill-Parot, qui est au cœur de son chapitre (p. 227-268). Il s'intéresse précisément au transfert de textes de magie talismanique et astrale de l'Orient vers l'Occident, notamment à partir de traductions arabo-latines des $\mathrm{XII}^{\mathrm{e}}$-XIII ${ }^{\mathrm{e}}$ siècles. Il met ainsi en scène les théologiens, les 
philosophes et bien sûr les magiciens, pour mieux décrire les modalités et les enjeux de la réception de ces textes chez les Latins. $\mathrm{N}$. Weill-Parot livre une très belle conclusion pour le refus, dans le cas de la magie astrale orientale, du concept d' " acculturation " ou d'" acculturation philosophique" tel qu'il est présenté par Alain de Libera ${ }^{3}$. Il propose plutôt le modèle du canevas : "Le texte d'origine arabe a été élaboré dans un contexte culturel donné qui lui donne un sens précis. [...] Une fois transmis dans une autre culture et une civilisation différente, le texte acquiert par son étrangeté même un indéniable pouvoir » (p. 266-267). Godefroid de Callataÿ s'interroge, quant à lui, sur les lecteurs des Rasā'il Ikhwān al-Ṣafă’ [Épîtres des Frères de la Pureté], corpus de nature encyclopédique dont l'influence est indéniable dans les communautés médiévales aussi bien musulmanes, juives que chrétiennes (p. 269-302). À partir de cinq thèmes l'ismaélisme, le néoplatonisme, l'ésotérisme, l'allégorisme et l'encyclopédisme -, G. de Callataÿ donne une première identification des catégories de personnes et des milieux culturels qui reçoivent le corpus ikhwānien. Il obtient ainsi quelques éléments sur les raisons et les modalités de circulation de ce corpus non orthodoxe dans les pays d'Islam et au-delà de leurs frontières. Le cas du monde latin est intéressant, car l'étude de la transmission indirecte se révèle strictement nécessaire. En effet, si l'historien se limitait aux traductions arabo-latines, il serait amené à sous-estimer l'impact réel des Frères de la pureté. Mohammad Bagheri rédige ensuite un court chapitre, qui aurait pu être inséré dans la partie sur les "sciences pratiques", montrant les compétences mathématiques que Kūshyār ibn Labbān ( $\mathrm{m}$. avant 1047) démontre indéniablement dans son ouvrage astronomique al-zij al-jāmi' [le zij compréhensible] (p. 303-310). Il est regrettable que l'auteur n'ait apporté ici aucun argument concernant l'impact de cet ouvrage, même s'il mentionne qu'il est "l'un des travaux astronomiques les plus importants de la civilisation islamique, qui a influencé par la suite le développement de l'astronomie en Europe à travers l'Afrique du Nord et le Sud de l'Espagne (alAndalus) » (p. 309). Kūshyār ibn Labbān occupe aussi une place de choix dans le dernier chapitre de cette seconde partie, mais cette fois-ci pour son ouvrage sur l'astrologie horoscopique intitulé al-madkhal fi șinā' at ahkām al-nujūm [L'introduction à l'astrologie]. Yunli Shi et Haohao Zhu détaillent l'importance (jusqu'au XVIII siècle, au moins) de sa traduction chinoise réalisée au XIV ${ }^{\mathrm{e}}$ siècle (p. 311-335). À partir de l'analyse des travaux de Xue Fengzuo (m.1680), importante figure de l'introduction de l'astrologie européenne en Chine au XVII ${ }^{e}$ siècle, ils montrent que l'érudit confucianiste a su intégrer des savoirs astrologiques islamiques aux nouveaux savoirs européens arrivés en Chine.

6 La troisième et dernière partie de l'ouvrage sous recension s'intitule « réception et organisation de la connaissance ». Iolanda Ventura débute cette partie avec une très belle contribution sur les sources éventuelles de la célèbre compilation de textes pharmaceutiques connue par son incipit Circa instans et attribuée au médecin de Salerne Matthaeus Platearius (m.ca 1161) (p.339-401). Pour cela, elle s'intéresse en particulier à deux remèdes largement utilisés dans l'Antiquité et au Moyen Âge: l'absinthe et le musc. I. Ventura souhaite proposer une histoire de la composition des textes et les chemins possibles de transmission et d'information à travers les sources disponibles. De sa connaissance approfondie des textes, elle conclut entre autres que la description de «Salerne comme un milieu dans lequel les traditions constantinienne, dioscoridienne et locale fusionnaient sans difficultés apparentes est brisée. Le Circa instans n'est pas un simple creuset, mais bien le miroir d'un conflit de traditions compliqué » (p.398). Véronique Boudon-Millot poursuit en interrogeant l'apport des 
traducteurs arabes dans le débat sur l'authenticité des traités médicaux attribués à Galien de Pergame (m.ca. 216) (p.403-423). Elle revient d'abord sur les critères qui amènent Galien lui-même à juger de l'authenticité des traités hippocratiques, avant de présenter ceux, inspirés des précédents, du célèbre traducteur et médecin chrétien nestorien Hunayn ibn Isḥāq $(\mathrm{m}$. 873) à propos des traités galéniques. En prenant l'exemple de quatre de ces traités (deux perdus et deux pour lesquels les débats sur l'authenticité sont encore d'actualité), V. Boudon-Millot mesure la précision des critères d'Hunayn et la manière avec laquelle les philologues modernes les considèrent. En conclusion, ils seraient «relativement sûrs et finalement assez peu éloignés des nôtres » (p. 422). Jean-Marc Mandosio centre son chapitre sur Pierre de Zélande ( $\mathrm{xv}^{\mathrm{e}}$ siècle) et son Lucidarius de rebus mirabilibus [Élucidation des choses merveilleuses] (p. 425-456). Il s'intéresse en particulier à une de ses parties, le Compendium de radiis, qui n'est autre qu'une paraphrase du texte éponyme dû au " philosophe des Arabes » alKindī (m. 873). L'importance historique du Lucidarius ne réside pas dans sa diffusion personne ne semble l'avoir lu (p. 428) -, mais dans le projet de son auteur. L'objectif de J.-M. Mandosio est donc de nous convaincre de tout l'intérêt de ce projet en s'appuyant notamment sur les raisons de la reprise commentée (avec des références à la Bible et à saint Jérôme) du texte d'al-Kindī, alors même qu'il est condamné à la fin du xiII ${ }^{\mathrm{e}}$ siècle par Gilles de Rome dans ses Errores philosophorum et qu'il ne jouit pas d'une bonne réputation.

7 En guise d'épilogue, Michio Yano (p. 457-473) et Agostino Paravicini Bagliani (p. 475-480) concluent brièvement des perspectives orientales et occidentales des travaux présentés. En plus de donner quelques exemples d'échanges en astrologie entre les pays d'Islam, l'Inde et la Chine, M. Yano en profite pour questionner les véritables frontières entre l'Est et l'Ouest.

8 L'ensemble des contributions regroupées dans ce volume offre une belle étude de "l'impact que constitua [...] l'introduction massive de textes arabes, recherchés pour le savoir global qu'ils véhiculaient, que celui-ci fût novateur ou non par rapport à la science grecque » (p. 20). En ce qui concerne l'Europe latine ou vernaculaire, les enjeux de l'ouvrage sont sans conteste amplement relevés par les différents auteurs. Plusieurs disciplines sont ainsi illustrées avec, en particulier, l'astrologie, la magie, l'algèbre, la mécanique, l'astronomie, la médecine, la pharmacopée ou encore l'alchimie. En revanche, même si l'impact des sciences arabes en Asie est central dans plusieurs contributions, il reste beaucoup moins étudié que celui en Europe. On pourrait regretter un tel déséquilibre, mais je n'y vois, pour ma part, qu'une photographie des recherches actuellement menées en histoire des sciences arabes. Cet ouvrage se révèle donc fort utile à plus d'un titre pour notre communauté; d'abord dans sa démonstration des questions de recherche qui se posent encore et toujours pour l'appropriation des sciences des pays d'Islam en Europe, puis dans ses encouragements pour des recherches en lien avec l'Asie. 


\section{NOTES}

1. Deux articles récents viennent compléter cette synthèse à propos de l'algèbre médiévale latine : M. MOYON, « The Liber Restauracionis : A newly discovered copy of a mediæval algebra in Florence", Historia Mathematica, 45/4 (2018), [en ligne]: https://doi.org/10.1016/j.hm. 2018.07.001; ID., «La restauration et la comparaison, ou l'art de résoudre des équations quadratiques dans l'Europe latine ", Revue d'histoire des mathématiques, 23/2 (2017), p. 233-299.

2. Il n'est pas aisé de se livrer à une étude de ce genre tellement les corpus manuscrits sont souvent bien maigres, surtout pour les périodes anciennes comme le Moyen Âge. C'est sans doute pour cela, comme le déplore D. Juste, qu' «aucune évaluation systématique de l'impact des sources arabes sur aucun domaine de la connaissance en Europe n'a [encore] été menée » (p. 173). Nous avons réalisé, quant à nous, une telle étude, pour les mathématiques et l'astronomie, concernant les manuscrits arabes subsahariens aujourd'hui disponibles dans les bibliothèques sahéliennes. En effet, nous avons jugé ce corpus de taille suffisamment grande pour le faire et nous avons ainsi pu établir quelques éléments caractéristiques des pratiques scientifiques de l'Afrique subsaharienne héritées des textes arabes : M. MOYoN, « Mathématiques et astronomie dans les "manuscrits du désert" : première approche", dans Kh. KCHIR (éd.), Revisiter l'histoire des sciences, des savoirs, des techniques et des arts au Moyen Âge, Tunis, à paraître ; ID., "Scientific Practices in Sub-Saharan Africa », dans S. BRENTJES (éd.), Routledge Handbook on Science in the Islamicate World: Practices From the 8th to the 19th Century, Londres, à paraitre.

3. Voir, en particulier, Alain de LIBERA, Penser au Moyen Âge, Paris, 1991, p. 109-116. Plus récemment: ID., "Les latins parlent aux latins ", dans P. BÜTTGEN, A. DE LIBERA, M. RASHED et I. ROSIER CATACH (dir.), Les Grecs, les Arabes et nous. Enquête sur l'islamophobie ambiante, Paris, 2009, p. $175-215$.

\section{AUTEURS}

\section{MARC MOYON}

Université de Limoges 